\title{
Fine-Needle Aspiration of Spinal Osteoblastoma in a Patient With Lymphangiomatosis
}

Michael G. Rhode, M.D., David R. Lucas, M.D., Cynthia H. Krueger, M.D., and Robert T. Pu, M.D., Ph.D.*

\begin{abstract}
Diagnosis of osteoblastoma by fine-needle aspiration (FNA) is rare, as in most patients, excisional biopsy is performed. We report a case of FNA diagnosis of a spinal osteoblastoma in a lymphangiomatosis patient. The patient had a history of lymphangiomatosis since birth with lymphangiomas removed from various locations, and had radiograph evidence of multifocal vertebral involvement. He presented with an enlarging C3 posterior element vertebral lesion. Clinically and radiologically, it was suspicious for lymphangiomatosis involvement. A computed tomography (CT)-guided FNA yielded a moderately cellular specimen. Lesional cells exhibited plasmacytoid features, fine chromatin, smooth nuclear membranes, and binucleation, in a background of occasional osteoclastic cells, spindle cells, and osteoid matrix. A cytological diagnosis of osteoblastoma was favored and confirmed with follow-up surgical biopsy. We believe that this is the first case reporting an osteoblastoma diagnosed by FNA in a lymphangiomatosis patient. We describe the clinical and cytological findings of osteoblastoma and its differential diagnosis. Diagn. Cytopathol. 2006;34:295-297. o 2006 Wiley-Liss, Inc.
\end{abstract}

Key Words: fine-needle aspiration; osteoblastoma; lymphangiomatosis

Osteoblastoma (OB) is a benign tumor representing less than one percent of all primary bone tumors and over three percent of benign primary tumors. It has an affinity for young males primarily in the second decade. The tumor most commonly involves the vertebral column, particularly the posterior or dorsal elements, as well as the sacrum. It also occurs in the long bones (with a slight metaphyseal over diaphyseal predominance), jaw, and craniofacial bones; however, any bone can be affected. ${ }^{1,2}$ Symptoms consist of dull, progressive bone pain that is more generalized than that of osteoid osteoma, and less likely to be relieved by aspirin or other analgesics. Rarely, systemic

Department of Pathology, University of Michigan Medical School, Ann Arbor, Michigan

*Correspondence to: Robert T. Pu, M.D., Ph.D., Department of Pathology, University of Michigan Hospitals, 1500 E. Medical Center Drive, Room 2G332, Ann Arbor, MI 48109. E-mail: robertpu@umich.edu

Received 28 June 2005; Accepted 6 October 2005

DOI 10.1002/dc.20436

Published online in Wiley InterScience (www.interscience.wiley.com). symptoms can occur, including weight loss, fever, and anemia. ${ }^{3}$ Spinal lesions may present with neurologic symptoms as a result of mechanical compression, as well as scoliosis. ${ }^{1,4}$ Because of its capacity for progressive growth with local bony destruction creating clinical symptoms as well as a potential for aggressive behavior, complete surgical excision is the preferred treatment. ${ }^{5,6}$ Osteoblastoma is not known to be associated with any syndrome.

\section{Case Report}

A 15-yr-old male with a significant past medical history of diffuse lymphangiomatosis presented for evaluation of neck pain that was slowly progressing over the past year. The pain was described as sharp, and worsened with activity but relented with ibuprofen. There was occasional concomitant right upper arm numbness and tingling without weakness. No other neurological symptoms were present. Physical examination showed tenderness to palpation of the right neck without an apparent mass. A complete neurological examination was normal.

His past medical history was notable for diffuse lymphangiomatosis at birth, and numerous lymphangiomas removed from various locations in the thorax and extremities over the years, including the mediastinum, skull, humerus, tibia, femurs, and pelvic region. Clinical and radiological examinations were notable for several intraabdominal and thoracic lesions. Multiple lytic lesions in the thoracic and lumbar spine involving the vertebral bodies were noted as well. He received treatment with $\alpha-2$ Interferon, with stabilization of his lymphangiomas several years before his latest presentation.

An initial CT scan 6 mo earlier revealed a $0.9 \mathrm{~cm}$ enhancing soft tissue focus with no aggressive features (believed to represent a lymph node), associated with the C3 vertebral body on the right side. This mass progressed to a $2.9 \mathrm{~cm}$ lesion on a follow-up scan 5 mo later. The latter CT showed an expansile lytic mass arising from the lateral posterior elements at $\mathrm{C} 3$ with evidence of bony irregularity, poorly defined margins, and infiltration into the ver- 


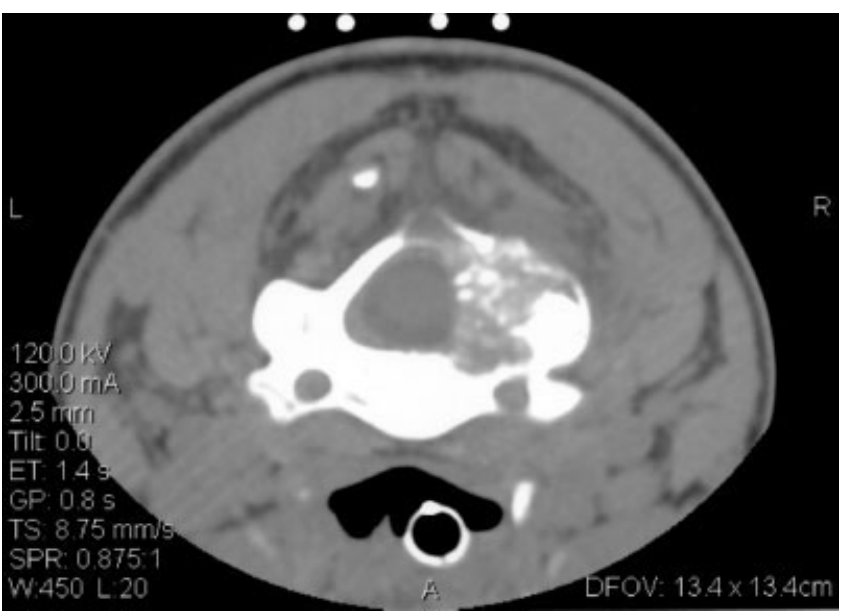

Fig. 1. CT scan image. It discloses an expansile lytic bone tumor arising from the left lateral posterior elements, with aggressive features, including secondary involvement of the vertebral body.

tebral body (Fig. 1). On MRI, the mass was homogeneous and had a T1 signal slightly higher than the muscle with an intermediate-to-high T2 signal. A clinical working diagnosis of recurrent lymphangiomatosis with concern for degeneration into a more aggressive process was made.

A fine-needle aspiration (FNA) was performed showing a moderately cellular specimen in a background of blood. The cells exhibited plasmacytoid features with round nuclei having somewhat fine chromatin and smooth nuclear membranes, and distinct, single nucleoli. There was a moderate amount of pale-blue to blue cytoplasm on Papanicolaou and Diff Quick stains, respectively, with a perinuclear "Hof" and peripheral cytoplasmic condensation (Fig. 2A). Occasionally, these cells were binucleated. Benign appearing osteoclastic giant cells and spindle cells were present in small number (Fig. 2B and C). The cell block revealed cellular groups, with an eosinophilic matrix interspersed between the cells consistent with osteoid (Fig. 2D). A cytologic diagnosis of an osteoblastic neoplasm, favoring osteoblastoma, was made. Excision of the tumor with C2-3 fusion was performed. Histologic diagnosis confirmed osteoblastoma (Fig. 3).

\section{Discussion}

We report a case of FNA diagnosis of OB in a lymphangiomatosis patient with a spinal lesion. In fact, the clinical and radiological impressions favored recurrent lymphangiomatosis. CT scans for lymphangiomas in bone are widely varied typically revealing a single, often multiloculated, sharply delineated, cystic/solid mass with a uniform, homogeneous appearance having no periosteal reaction in adjacent bone. ${ }^{7}$ However, the radiologic appearance can exhibit irregular cyst walls with calcification, as well as inhomogeneous patterns leading to a broad differential diagnosis. ${ }^{8}$ Such a differential diagnosis includes tumors of vascular origin, aneurysmal bone cyst, giant cell tumor,

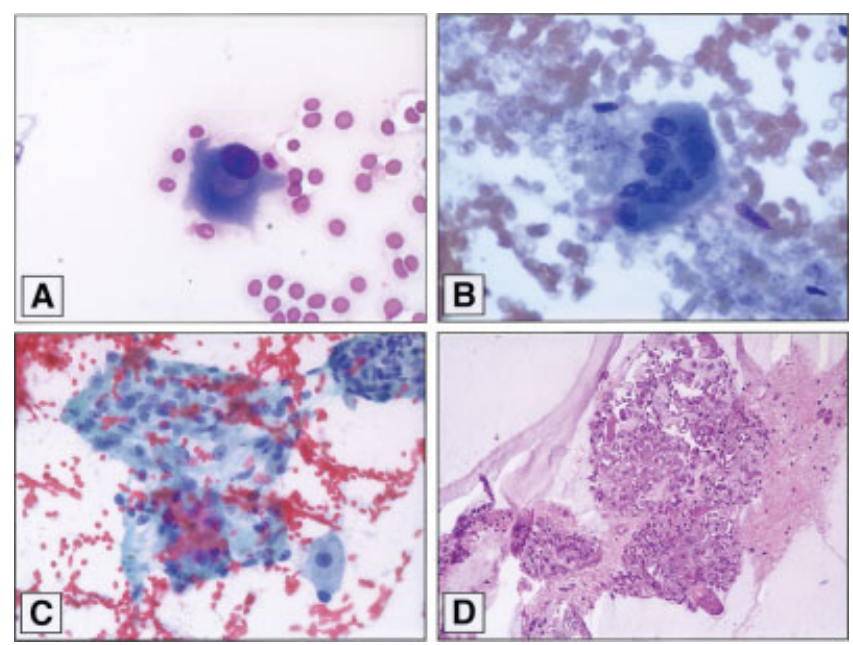

Fig. 2. Cytology smears. A and B: Plasmacytoid appearance of osteoblast and multinucleated osteoclast cells (Diff-Quik, $\times 400$ ), C: Osteoblasts and banal spindled cells (Papanicolaou, $\times 400$ ), and D: Cell block exhibiting osteoid with osteoblasts $(\mathrm{H} \& \mathrm{E}, \times 100)$. [Color figure can be viewed in the online issue, which is available at www.interscience.wiley.com.]

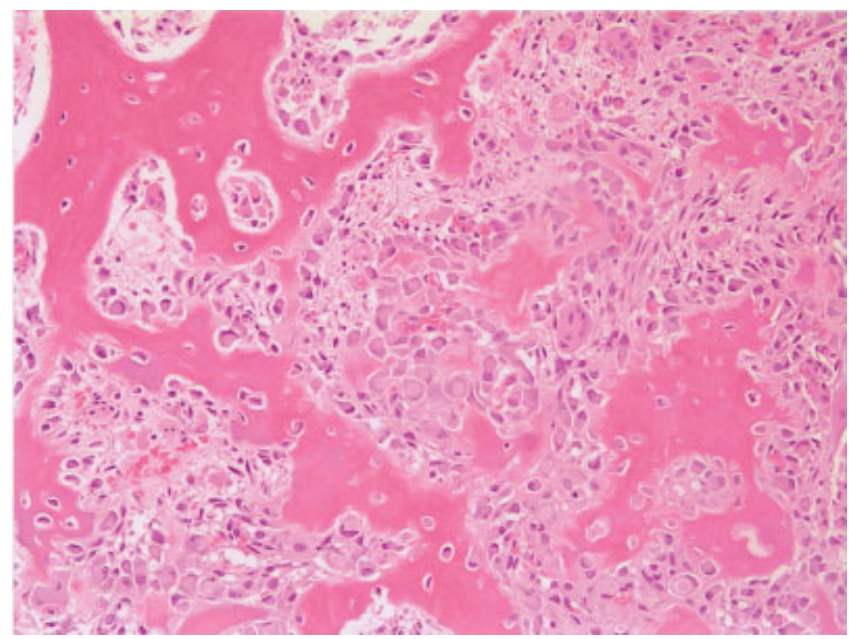

Fig. 3. Histology of the tumor. It demonstrates the classic features of OB including interanastomosing trabeculae of woven bone rimed by a single layer of osteoblasts, and loose fibrovascular stroma. (H\&E, $\times 200)$. [Color figure can be viewed in the online issue, which is available at www. interscience.wiley.com.]

fibrous dysplasia, chondroma or chondrosarcoma, as well as osteoblastoma and osteosarcoma. ${ }^{9}$

The FNA findings in this case were consistent with an osteoblastoma as opposed to the findings of cystic fluid, with lymphocytes and histiocytes in lymphangioma. Confirmation of the diagnosis was made by follow-up excisional biopsy. We believe that this is the first reported case of the osteoblastoma in a lymphangiomatosis patient. FNA provided a diagnosis leading to a different clinical treatment and management. The significance of association of osteoblastoma and lymphangiomatosis is not clear. One could speculate that there may be an etiologic relation. For 
example, it has been proposed that osteoid osteoma (OO), a tumor with very similar morphologic features with osteoblastoma, may derive from an aberrant vascular development. $^{10}$

Osteoblastoma was originally described in 1932 by Jaffe and Mayer. ${ }^{1}$ The age range for osteoblastoma is wide, encompassing those as young as 5 to those 45 or even $75 \mathrm{yr}$ of age. ${ }^{6} \mathrm{OB}$ occurs within the bone cortex in $\sim 2 / 3$ of the cases, with an average size of $3.2 \mathrm{~cm}$ on radiographic studies, slightly larger when it involves vertebra for which it has a predilection to affect. ${ }^{11}$ Expansion of adjacent bone is seen in over $70 \%$ with reactive sclerosis seen in a lesser majority. ${ }^{1}$ Particular to spinal OB is that it is more apt to behave in an aggressive manner with cortical destruction as well as extend into neighboring soft tissues, a facet not seen as often in its long bone variant. ${ }^{1,2}$

Histologically, there are anastomosing bony trabeculae of osteoid and/or woven bone lined by a single layer of osteoblasts. Infrequently, these cells occur in small clusters. A loose fibrovascular stroma consisting of capillaries and groups of spindle cells lies between the lined trabecular portions of the tumor. There is variable mineralization and the border is sharply circumscribed with no or little permeation between pre-existing trabeculae (differentiating it from osteosarcoma). The pushing edge of the tumor exhibits maturation as it contacts the normal adjacent bony tissue. ${ }^{1,6,12}$ A minority of cases express occasional bizarre multinucleated pleomorphic cells with smudged chromatin giving a degenerated appearance. ${ }^{1,3}$ Epithelioid osteoblasts are generally not prominent in most tumors. ${ }^{1}$

FNA cytological description of OB has been rare, but it usually is a moderately cellular specimen with mixed cell types. ${ }^{12,13}$ There are numerous plasmacytoid osteoblasts of various sizes with eccentric rounded nuclei, multinucleated osteoclastic giant cells, groups of benign appearing spindle cells, and amorphous, osteoid matrix sometimes lined by osteoblasts. Mitotic activity is not characteristic and no abnormal forms should be present.

The differential diagnosis of $\mathrm{OB}$ mainly involves $\mathrm{OO}$ and osteosarcoma (OS). OB and OO are often microscopically indistinguishable; clinical and radiographic parameters may be of assistance in such situations. ${ }^{1}$ With regard to differentiating $\mathrm{OB}$ from OS, the most important finding is the destructive permeation between existing trabeculae and surrounding tissue seen in OS. The intervening stroma of OS is also denser, consisting of compact cellularity compared to the loose aggregates found in OB. On cytology, OS also has greater nuclear hyperchromasia, atypia as well as mitotic activity with necrosis often observed.
From a practice point of view, FNA shows good to excellent correlations to histological sections. When compared to radiographic accuracies, FNA compares favorably suggesting FNA can be of value in the standard diagnostic workup of a bony tumor, especially when other clinical or radiographic parameters do not agree. ${ }^{14,15}$

In summary, we believe this is the first case of osteoblastoma discovered in association with lymphangiomatosis. Whether a causative relationship is present is not known; however, the possibility of a vascular link in osteoid osteoma etiology as speculated in the literature may provide insight to the relationship between the two conditions.

\section{References}

1. Lucas DR, Unni KK, McLeod RA, et al. Osteoblastoma: clinicopathologic study of 306 cases. Hum Pathol 1994;25:117-134.

2. Papagelopoulos PJ, Galanis EC, Sim FH, et al. Clinicopathologic features, diagnosis, and treatment of osteoblastoma. Orthopedics 1999;22: 244-247; quiz 248-249.

3. Mirra JM, Cove K, Theros E, et al. A case of osteoblastoma associated with severe systemic toxicity. Am J Surg Pathol 1979;3:463-471.

4. Ozaki T, Liljenqvist U, Hillmann A, et al. Osteoid osteoma and osteoblastoma of the spine: experiences with 22 patients. Clin Orthop Relat Res 2002;397:394-402.

5. Gitelis S, Schajowicz F. Osteoid osteoma and osteoblastoma. Orthop Clin North Am 1989;20:313-325.

6. Unni KK, Dahlin DC. Dahlin's bone tumors: general aspects and data on 11,087 cases. Philadelphia: Lippincott-Raven; 1996. p 463.

7. Mendez JA, Hochmuth A, Boetefuer IC, et al. Radiologic appearance of a rare primary vertebral lymphangioma. AJNR Am J Neuroradiol 2002;23:1665-1668.

8. Davidson AJ, Hartman DS. Lymphangioma of the retroperitoneum: CT and sonographic characteristic. Radiology 1990;175: 507-510.

9. Keenen TL, Buehler KC, Campbell JR. Solitary lymphangioma of the spine. Spine 1995;20:102-105.

10. Lavrishcheva GI. [The essence of osteoid osteomas and osteoblastomas]. Arkh Patol 1991;53:24-28.

11. Malcolm A, Schiller A, Schneider-Stock R. Osteoblastoma. In: Fletcher CDM, Unni KK, Mertens F, editors. Pathology and genetics of tumours of soft tissue and bone. Lyon: IARC Press; 2002. p 262 263

12. Walaas L, Kindblom LG. Light and electron microscopic examination of fine-needle aspirates in the preoperative diagnosis of osteogenic tumors: a study of 21 osteosarcomas and two osteoblastomas. Diagn Cytopathol 1990;6:27-38.

13. Layfield LJ. Cytopathology of bone and soft tissue tumors. New York: Oxford University Press; 2002. p 266.

14. Bommer KK, Ramzy I, Mody D. Fine-needle aspiration biopsy in the diagnosis and management of bone lesions: a study of 450 cases. Cancer 1997;81:148-156.

15. Layfield LJ, Armstrong K, Zaleski S, et al. Diagnostic accuracy and clinical utility of fine-needle aspiration cytology in the diagnosis of clinically primary bone lesions. Diagn Cytopathol 1993;9: $168-173$. 